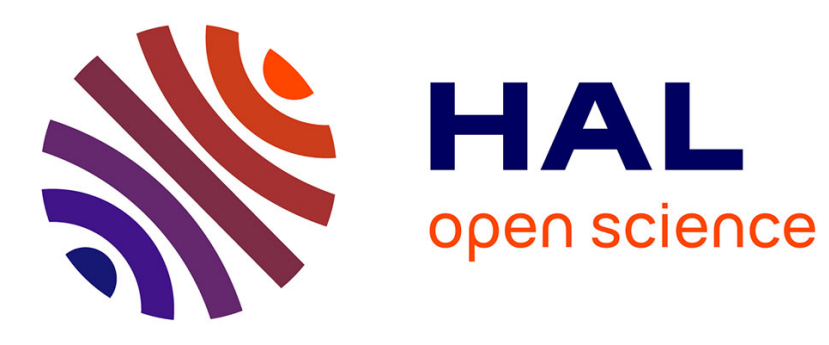

\title{
Whistler mode waves at magnetotail dipolarization fronts
}

H. Viberg, Y. V. Khotyaintsev, A. Vaivads, M. André, H.S. Fu, Nicole Cornilleau-Wehrlin

\section{- To cite this version:}

H. Viberg, Y. V. Khotyaintsev, A. Vaivads, M. André, H.S. Fu, et al.. Whistler mode waves at magnetotail dipolarization fronts. Journal of Geophysical Research Space Physics, 2014, 119, pp.26052611. 10.1002/2014JA019892 . hal-01552032

\section{HAL Id: hal-01552032 \\ https://hal.science/hal-01552032}

Submitted on 11 Oct 2021

HAL is a multi-disciplinary open access archive for the deposit and dissemination of scientific research documents, whether they are published or not. The documents may come from teaching and research institutions in France or abroad, or from public or private research centers.
L'archive ouverte pluridisciplinaire HAL, est destinée au dépôt et à la diffusion de documents scientifiques de niveau recherche, publiés ou non, émanant des établissements d'enseignement et de recherche français ou étrangers, des laboratoires publics ou privés.

$$
\text { Copyright }
$$




\section{Journal of Geophysical Research: Space Physics}

\author{
RESEARCH ARTICLE \\ 10.1002/2014JA019892 \\ Key Points: \\ - Statistics of whistler mode waves near \\ magnetotail dipolarization fronts \\ -Whistlers are much more common \\ near DFs than at any place in \\ the magnetotail \\ - Electron pitch angle distributions \\ consistent with betatron acceleration
}

Correspondence to:

H. Viberg,

henrik.viberg@irfu.se

\section{Citation:}

Viberg, H., Y. V. Khotyaintsev, A. Vaivads, M. André, H. S. Fu, and N. Cornilleau-Wehrlin (2014), Whistler mode waves at magnetotail dipolarization fronts, J. Geophys. Res. Space Physics, 119, 2605-2611, doi:10.1002/2014JA019892.

Received 15 FEB 2014 Accepted 18 MAR 2014 Accepted article online 20 MAR 2014 Published online 8 APR 2014

\section{Whistler mode waves at magnetotail dipolarization fronts}

\author{
H. Viberg ${ }^{1,2}$, Yu. V. Khotyaintsev ${ }^{1}$, A. Vaivads ${ }^{1}$, M. André ${ }^{1}$, H. S. Fu ${ }^{3}$, and N. Cornilleau-Wehrlin ${ }^{4,5}$ \\ ${ }^{1}$ Swedish Institute of Space Physics, Uppsala, Sweden, ${ }^{2}$ Department of Physics and Astronomy, Uppsala University, \\ Uppsala, Sweden, ${ }^{3}$ Space Science Institute, School of Astronautics, Beihang University, Beijing, China, ${ }^{4}$ LPP, Ecole \\ Polytechnique, CNRS, Palaiseau, France, ${ }^{5}$ LESIA, Observatoire de Paris, Meudon, France
}

Abstract We report the statistics of whistler mode waves observed in relation to dipolarization fronts (DFs) in Earth's magnetotail using data from the four Cluster spacecraft spanning a period of 9 years, 2001-2009. We show that whistler mode waves are common in a vicinity of DFs: between 30 and $60 \%$ of all DFs are associated with whistlers. Whistlers are about 7 times more likely to be observed near a DF than at any random location in the magnetotail. Therefore, whistlers are a characteristic signature of DFs. We find that whistlers are most often detected in the flux pileup region (FPR) following the DF, close to the center of the current sheet $\left(B_{x} \sim 0\right)$ and in association with anisotropic electron distributions $\left(T_{\perp}>T_{\|}\right)$. This suggests that we typically observe emissions in the source region where they are generated by the anisotropic electrons produced by the betatron process inside the FPR.

\section{Introduction}

Dipolarization fronts, DFs, are tangential discontinuities in the magnetotail, separating the plasma sheet and fast plasma flows, which can be created by various mechanisms such as magnetic reconnection [Sitnov et al., 2009; Fu et al., 2012c] and kinetic interchange instability [Pritchett and Coroniti, 2011]. DFs are identified by a sharp increase of $B_{z}$ GSM and are associated with electron and ion acceleration [Asano et al., 2010; Zhou et al., 2010; Fu et al., 2011] as well as various wave activities, e.g., electron holes, whistler, lower hybrid, and electron cyclotron waves [Le Contel et al., 2009; Sergeev et al., 2009; Zhou et al., 2009; Deng et al., 2010; Khotyaintsev et al., 2011; Hwang et al., 2011].

Whistler mode waves are right-hand circularly polarized electromagnetic waves with frequencies ranging from above the lower hybrid frequency, $f_{\mathrm{LH}}$, to below the electron cyclotron frequency, $f_{\mathrm{ce}}$, and are commonly observed in the magnetotail. Whistlers observed in the magnetotail typically lie in a frequency range from 0.1 to $0.6 f_{\text {ce, }}$ [Liang et al., 2012] and have a mean amplitude of $1 \%$ of the background magnetic field [Zhang et al., 1999]. Bursts of whistler activities are often observed in connection with fast plasma flows [Liang et al., 2012].

A number of case studies [Le Contel et al., 2009; Deng et al., 2010; Khotyaintsev et al., 2011; Huang et al., 2012] reported observations of whistlers in relation to DFs. In particular, they found whistlers in the magnetic flux pileup region (FPR), where the electron distribution has a perpendicular anisotropy, which is large enough to drive whistlers via the whistler anisotropy instability [Le Contel et al., 2009]. Le Contel et al. [2009] also showed that the bandwidth of the whistler signal correlated with the anisotropy, i.e., higher anisotropy is associated with a larger bandwidth, and that the electron anisotropy decreases away from the equator so that the waves have a higher growth rate closer to the equator. Using data from four Cluster spacecraft, Khotyaintsev et al. [2011] show that the generation region is located at the equator. They suggested that whistlers provide evidence of betatron heating in the FPR, which creates a temperature anisotropy $T_{\perp}>T_{\|}$. Panov et al. [2013] showed that whistlers can efficiently scatter electrons in pitch angle (energies close to thermal energies, i.e., 1-5 keV), making the electron distribution more isotropic.

Despite existing case studies, the statistical relation between DFs and whistlers has not been established. If such a relation exists, it could be used as a signature of DF/transient reconnection in the magnetosphere. Planetary missions usually do not have particle data of sufficient resolution, so wave observations can then be used instead to infer the presence of DFs. In this letter we present statistics of whistlers in relation to DFs observed by Cluster [Escoubet et al., 1997] during 2001-2009. 


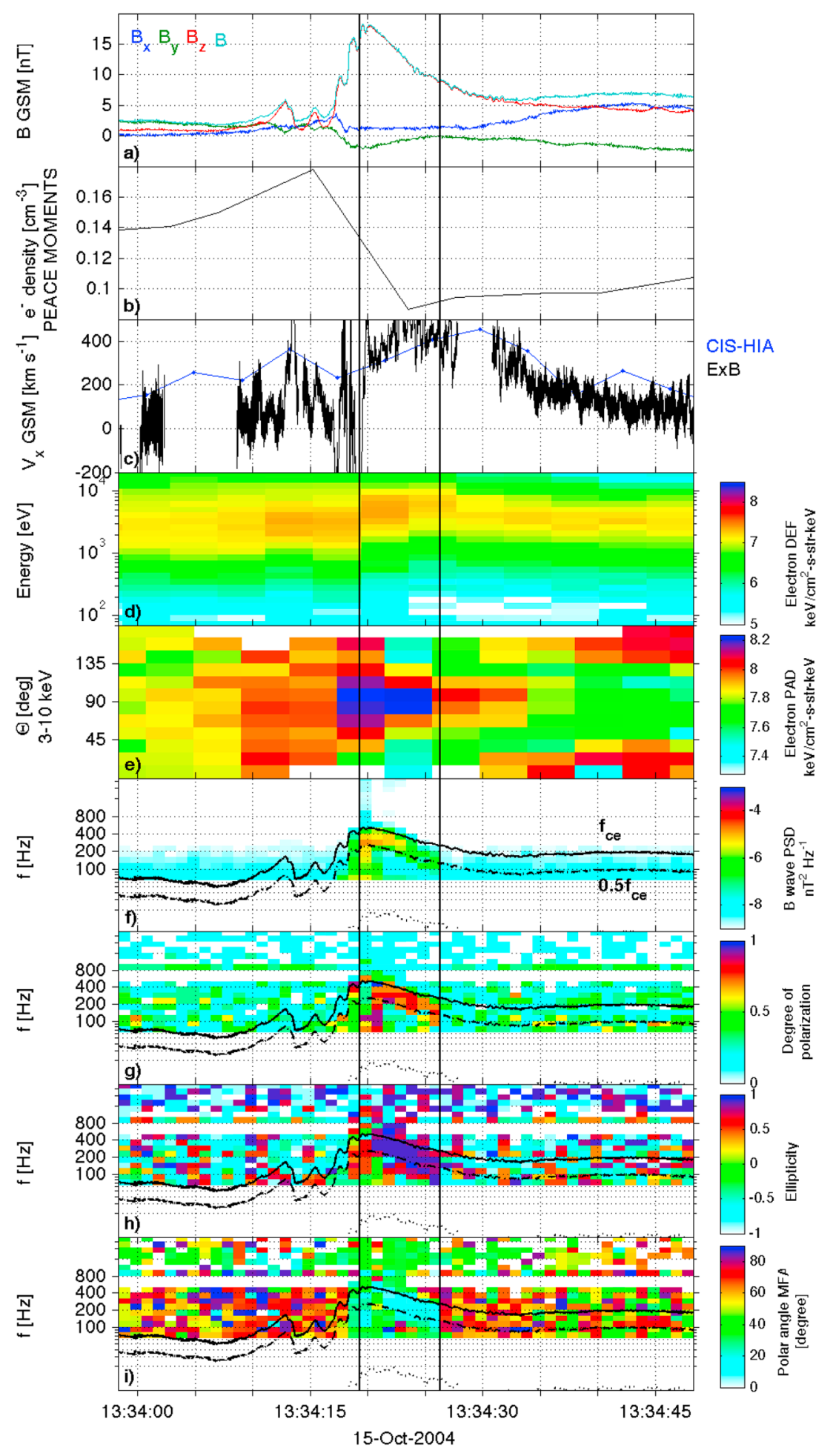

Figure 1. Overview of a DF event observed by C1. (a) Magnetic field from FluxGate Magnetometer (FGM) [Balogh et al., 2001], (b) electron density from PEACE [Owen et al., 2001], (c) ion velocity (X GSM) from both CIS [Rème et al., 2001] and $v_{\text {EXB }}$ using Electric Fields and Waves (EFW) [Gustafsson et al., 2001], (d) electron differential energy flux, (e) pitch angle distribution for energies of 3-10 keV, (f) power spectral density of the wave magnetic field, (g) the degree of polarization, (h) the ellipticity, and (i) the wave angle with respect to the magnetic field. Data for Figures $1 \mathrm{f}-1 \mathrm{i}$ are calculated from spectral matrices produced by onboard STAFF Spectrum Analyzer [Cornilleau-Wehrlin et al., 2003], using the PRASSADCO program [Santolik, 2000; Santolik et al., 2003]. The black lines in Figures $1 \mathrm{f}-1 \mathrm{i}$ show $f_{\text {ce }}$ and $0.5 f_{\text {ce, }}$, respectively. The black vertical lines mark the region where the strongest waves are observed. 


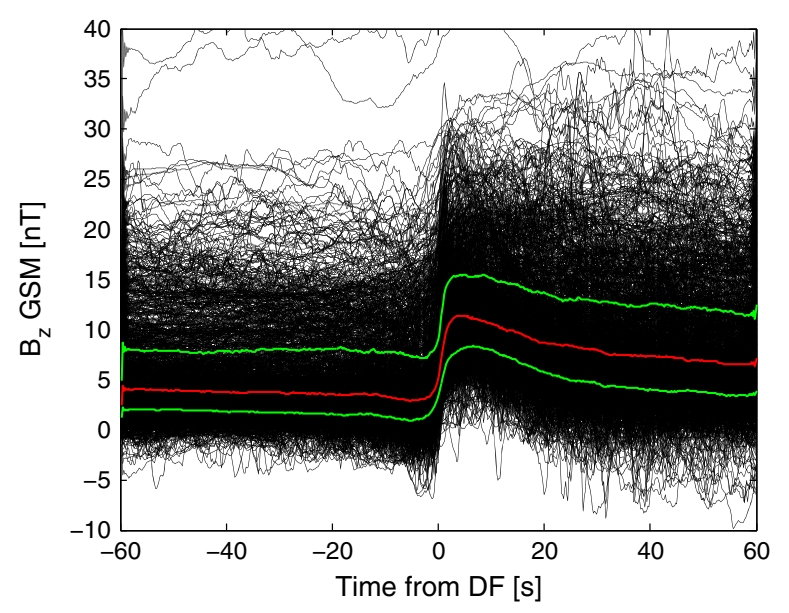

Figure 2. Superposed epoch of all DF events observed by the Cluster spacecraft. The red line shows the median $B_{z}$, and the two green lines the 25 th and 75 th percentiles. The median jump in $B_{z}$ is about $6.1 \mathrm{nT}$, and the median duration of the DF is about $1.8 \mathrm{~s}$.

\section{Event Study}

Figure 1 shows an example of a whistler wave event behind a DF. In this event, the spacecraft (SC) are located at $(-13,8,-0.2) R_{E}$ GSM. The DF is clearly seen as a sharp increase of $B_{z}$ (Figure 1a). The electron density (Figure $1 b$ ) decreases at the DF. Figure 1c shows the $x$ component of the ion velocities, using both data from the Cluster lon Spectrometry-Hot lon Analyzer instrument (blue) and the calculated $v_{\mathrm{E} \times \mathrm{B}}$ velocity, low pass filtered between 0 and $10 \mathrm{~Hz}$ (black). We see that $v_{x}$ increases in the FPR up to a maximum of $450 \mathrm{~km} / \mathrm{s}$. The velocity peak is located behind the DF, so in this case we are observing a growing flux pileup region [Fu et al., 2011]. The plasma flow velocity is not

high enough to cause significant Doppler shift of the measured frequency (maximum frequency shift is $5 \%$ ).

Figure 1d shows the electron differential energy flux (DEF) from Plasma Electron and Current Experiment (PEACE), averaged over pitch angle. In the FPR, the temperature of the electron population increases from about $3 \mathrm{keV}$ to $5 \mathrm{keV}$. After this, the temperature drops to the same value as before. The pitch angle distribution is displayed in Figure 1e for energies between 3 and $10 \mathrm{keV}$. As can be seen, the distribution is mostly isotropic up until the DF, where the perpendicular flux increases, corresponding to $T_{\perp}>T_{\|}$. This is consistent with betatron heating of electrons in the FPR due to the increase of the magnetic field.

In the FPR, where the electron distribution has high perpendicular anisotropy, we observe an increase of the power spectral density of the wave magnetic field (Figure 1f). The wave maximum activity is localized

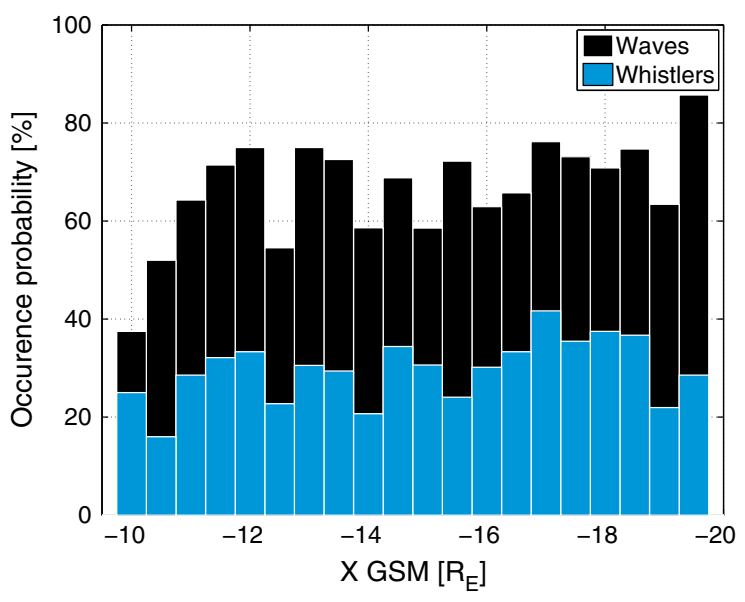

Figure 3. Distribution of waves and whistlers in $X$ (GSM). The height of the bar shows the ratio between the number of wave or whistler events, divided by the number of DF events, for each $X$ bin. The blue bars show the occurrence of any emission identified as a wave by our algorithm. The black bars show the occurrence of those emissions that were classified as whistlers. About $30 \%$ of the DFs are associated with whistlers, and about $60 \%$ with general waves. Since our algorithm probably rejects many whistlers, but accepts them as waves, the true whistler distribution would lie somewhere between the blue and black bars. between $f_{\text {ce }}$ and $0.5 f_{\text {ce }}$ (marked by black lines). The duration of the wave burst is about $8 \mathrm{~s}$. The time resolution of Spatio Temporal Analysis of Field Fluctuations (STAFF) Spectrum Analyzer (SA) is either $1 \mathrm{~s}$ (high bit rate) or $4 \mathrm{~s}$ (normal bit rate), both of which are much shorter than the typical duration of the FPRs. The median degree of polarization is $\sim 0.7$, and the median ellipticity is close to +1 for the whole burst (Figures $1 \mathrm{~g}$ and $1 \mathrm{~h}$ ). Such properties indicate that the wave belongs to the whistler mode. Figure 1i shows the wave angle with respect to the magnetic field. The median angle is $17^{\circ}$ for the whistler emission, showing that the wave propagates quasi-parallel to the magnetic field. Although we present only data from C1 in Figure 1, the same wave structure was observed by all four SC in this specific event; the characteristic spacecraft separation was $1100 \mathrm{~km}$ in GSM-X. The spectra in Figures $1 \mathrm{f}-1 \mathrm{i}$ are obtained from the STAFF SA instrument and are calculated onboard Cluster using the PRASSADCO algorithm [Santolik, 2000; Santolík et al., 2003]. 

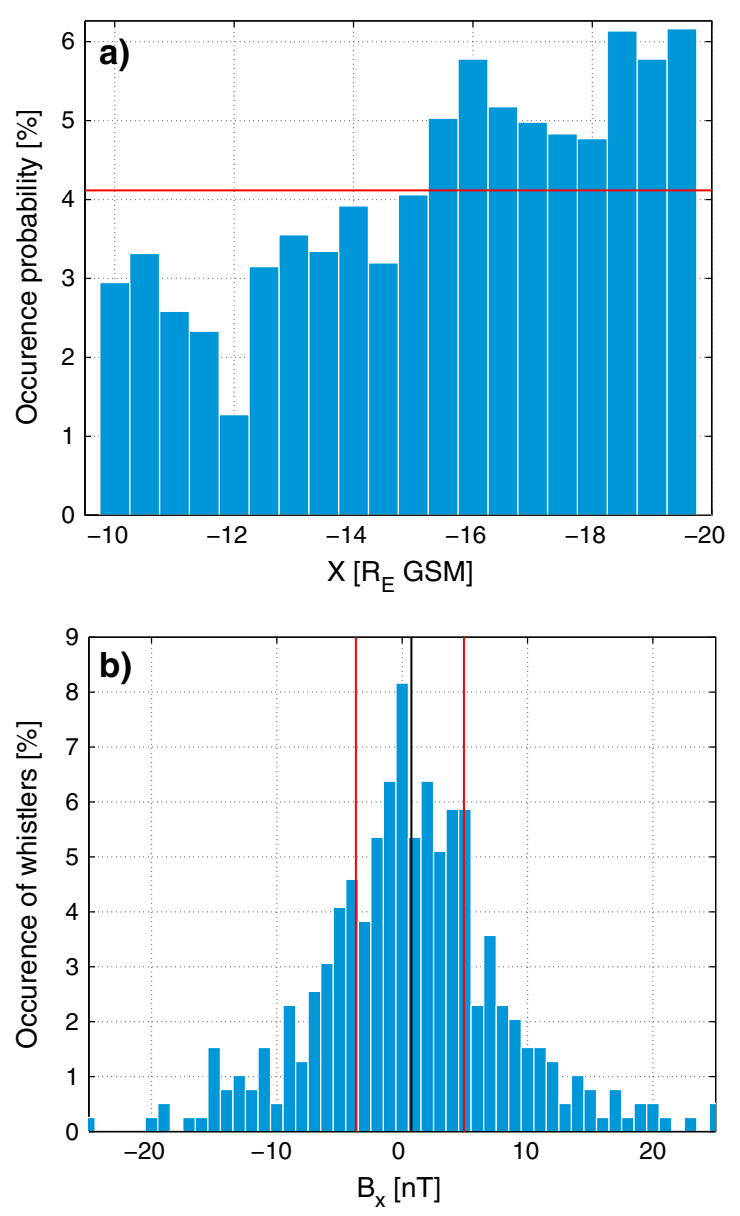

Figure 4. (a) Occurrence probability for finding whistlers anywhere in the tail box during the period from 2001 to 2009. We limit the search to the plasma sheet by using the criterion $\beta>0.5$. We do not use $\mathrm{C} 2$, since there is no CIS data available for that SC. The average probability is about $4 \%$. This is a significantly lower probability than for finding a whistler at a DF. (b) Occurrence probability versus $B_{x}$. The distribution is centered around $B_{x} \approx 0$ (black line), with $50 \%$ (red lines) of the cases lying within $\pm 5 \mathrm{nT}$, meaning the whistlers are mostly observed near the magnetic equator.

Jump of 6.1 nT and a median duration of 1.8 s. Our median flow

\section{Data Selection and Wave Identification}

We perform a statistical analysis of whistlers at DFs. Our study is done in two steps: first, we search for DFs in the magnetotail and then, from the events obtained from step one, identify whistlers.

\subsection{DF Observations}

We use the DF search algorithm by $\mathrm{Fu}$ et al. [2012a] but extend the search to all four SC, for the tail periods from 2001 to 2009; the tail box is defined as having $-20<X<-10 R_{E},-12<Y<12 R_{E}$, and $-5<Z<5 R_{E}$ (GSM). For all times where Cluster lon Spectrometry (CIS) data are available, the criterion $\beta>0.5$ is used to narrow the search. The DFs were identified by fitting a hyperbolic tangent function to $B_{z}$

$$
B_{\text {fit }}=\frac{a}{2} \tanh \left(\frac{\Delta t}{b / 2}\right)+\left(c+\frac{a}{2}\right),
$$

where $\Delta t=t-t_{\mathrm{DF}}$ is the time interval from $60 \mathrm{~s}$ before to $15 \mathrm{~s}$ after the DF. The fitting coefficients $a, b$, and $c$ are obtained for each event and represent the jump in $B_{z}$, the duration of the DF, and the magnitude of $B_{z}$ $1 \mathrm{~min}$ before the DF, respectively. The criteria on the coefficients are $a \geq 4 \mathrm{nT}, b \leq 8 \mathrm{~s}$, and $\sigma \leq 2.5 \mathrm{nT}$, where $\sigma$ is the standard error of the fitting. For the period 2001-2009, we found 1272 DF events, out of which 873 are unique, meaning that the 2 min DF intervals from different spacecraft do not overlap. $A$ superposed plot of all DFs for the tail epoch is shown in Figure 2. The red line shows the median $B_{z}$ and the two green lines show the 25th and 75th percentiles. We get a median jump of $6.1 \mathrm{nT}$ and a median duration of $1.8 \mathrm{~s}$. Our median flow velocity is $200 \mathrm{~km} \mathrm{~s}^{-1}$. The ion inertial length of each DF is calculated from ion moments where CIS data are available. We get a median ion inertial length of about $565 \mathrm{~km}$, which gives a median DF thickness of 0.64 ion inertial lengths. These results are consistent with Fu et al. [2012a].

\subsection{Whistler Wave Identification}

We search through the identified DF events for signs of whistlers by gradually filtering out nonwave signals, in a way similar to Bortnik et al. [2007]. We call a point in the spectra of Figure 1f-1h a "pixel," defined as having a certain time duration ( $1 \mathrm{~s}$ or $4 \mathrm{~s}$, depending on the STAFF SA operation mode) and a certain width in frequency. For all recognized DF events, defined as the period \pm 60 s from the DF, we first remove any pixel in the magnetic field spectrum that is less than 10 times the median at that specific frequency. We then look, for each time step, for local maxima in frequency in the remaining data and keep the strongest maxima and two pixels, one directly above and one directly below. The next step is to keep the pixels that are continuous in time, with the requirement that a signal must be at least two pixels wide in time. The final step is to look at each group of pixels and keep only those that have a median degree of polarization greater than 0.5 and a median ellipticity greater than 0.7 . The reason we use the median, rather than the mean, is that a noisy whistler signal is more likely to be accepted than if the mean had been used. The noisy nature 

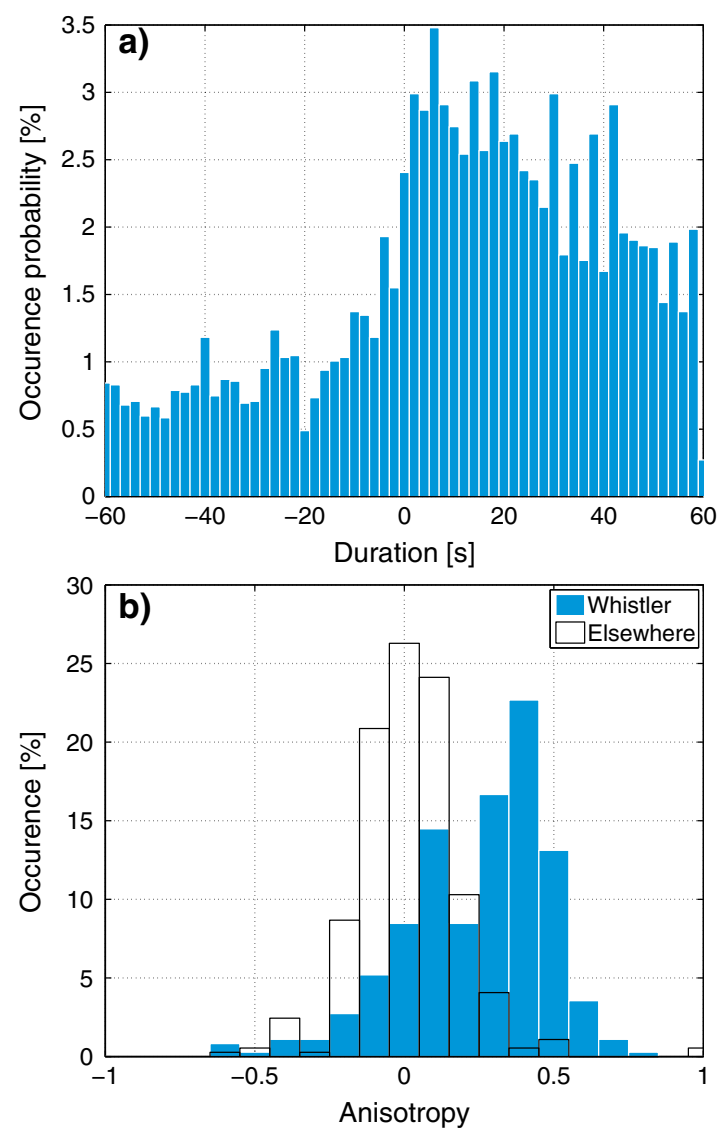

Figure 5. (a) Histogram of the time between the DF observation and the whistler emission. The plot is centered around the time of the DF observation. The whistlers are clearly more common at and after the DF than before. The probability begins to increase a few seconds before the DF. (b) Electron anisotropy during whistler emissions plotted against the anisotropy at the rest of each $2 \mathrm{~min}$ interval. The anisotropy is mostly $>0$ for whistlers, and it is also larger than elsewhere during the same event. This suggests that the whistlers are caused by the electron anisotropy. The vertical scale is the occurrence percentage for each group. of the ellipticity is clearly seen in Figure $1 \mathrm{~h}$. After all this is done, we have a list of identified 2 min DF events that contain whistler wave observations. We also keep a list of those events that exhibited general wave emissions, i.e., was sufficiently above median amplitude and had a distinct peak in the spectra but did not necessarily have the required length or contiguity.

The method most likely misses some whistlers due to the search criteria. For example, a wave identified as having the strongest local maximum in frequency at a specific time may turn out to not have the desired ellipticity and is then discarded. The second strongest peak of that event might have been a whistler wave. Using a certain number of pixels rather than a certain time to identify continuous wave signals is also likely to decrease the number of approved whistler events. Events lasting just a bit shorter than $8 \mathrm{~s}$ may be interpreted as only one data point in normal bit rate (time resolution $4 \mathrm{~s}$ ). Eighty-five percent of whistlers were observed in normal bit rate. Also, whistler emissions may be fragmented into many pieces, each shorter than two pixels, not fulfilling our criteria. Thus, we get a lower limit estimate of the occurrence rate of whistlers versus waves at DFs.

\section{Statistical Results}

Out of 1272 DF events, 859 were found to have wave emissions, and 394 events fulfilled the whistler criteria. The percentage of DFs that are associated with whistlers is thus somewhere between $31 \%$ to $67 \%$; see Figure 3. We note, however, that our DF detection algorithm is likely to miss many weaker or complex DFs. Since our algorithm has rather stringent conditions on what is deemed a whistler wave, we argue that the true percentage of DF events that exhibit whistler emissions is somewhere in between the ratios for whistlers and waves. The occurrence of both whistlers and DFs is lower closer to Earth, but the probability of observing a whistler once a DF is observed is approximately independent of distance along $X_{\mathrm{GSM}}$. This is consistent with the results of Fu et al. [2012b], who did not find a particular dependence of the anisotropy of energetic electron flux on the location of the DF. Also, the amplitude of observed whistlers shows no discernible dependence on distance (not shown). Most of the whistlers have small wave normal angles with respect to $B$ (angles below $35^{\circ}$ span $75 \%$ of the cases). Whistlers are mainly observed close to the magnetic equator, as can be seen in Figure $4 \mathrm{~b}$. The distribution of whistlers is centered around $B_{x} \approx 0$, with half of the observations lying within $\pm 5 \mathrm{nT}$. This tells us that the whistlers are mostly observed in the neutral sheet.

A histogram of the time difference from the DF to the whistler signal, Figure 5, shows that whistler observations are much more common after the DF than before, consistent with the waves being generated in the FPR, where the betatron acceleration is expected to create anisotropic temperature anisotropy [Khotyaintsev et al., 2011]. To study the electron anisotropy, we define factors $\alpha=T_{\perp} / T_{\|}-1$ for $T_{\perp}<T_{\|}$and $\alpha=1-T_{\|} / T_{\perp}$ for $T_{\perp}>T_{\|}$, which have values between -1 and +1 , where negative $\alpha$ means more parallel than perpendicular flux, and vice versa. In Figure 5b we plot distributions of $\alpha$ for two groups of events, one for the intervals 
when there are whistler emissions and one for when there are not. Clearly, almost all whistlers are associated with $\alpha>0$. For nonwhistler times, $\alpha$ is centered around 0 . Thus, we conclude that whistler emissions are caused by perpendicular electron temperature anisotropy resulting from betatron acceleration.

For comparison with DF-related whistlers, we searched for whistler in all 2 min intervals that Cluster spends in the tail box, with the criterion $\beta>0.5$ to limit the search to the plasma sheet. We found that the probability of detecting a whistler wave at a random position in the tail box is on average about $4 \%$; see Figure 4 . The probability of finding a whistler wave at a DF is thus about 7 times higher than for a random location in the magnetotail. The ratio of the total number of whistlers at DFs and the total number of whistlers in the tail box is about $8.4 \%$, so an observation of a whistler is not a definite indication of a DF. The minimum at around $-12 R_{E}$ is not statistically significant, as there are few DF events observed in this region giving large statistical error.

Almost all observed waves had a frequency less than $0.5 f_{\text {ce. }}$. The median frequency was about $0.16 f_{\text {ce, }}$, with 25th and 75th percentiles lying at $0.09 f_{\text {ce }}$ and $0.29 f_{\text {ce, }}$ respectively (not shown). This is consistent with the results presented by Zhang et al. [1999], who analyzed Geotail data consisting of approximately 1300 wave observations, though recorded throughout the magnetotail and not in any specific region or at any specific structure. The whistler wave in the event shown in Figure 1 is between $0.5 f_{\mathrm{ce}}$ and $f_{\mathrm{ce}}$, so it is not a whistler with typical frequency. Rather, we chose this particular event because it was observed by all four SC and had high resolution.

\section{Conclusions}

We present statistics of whistlers at dipolarization fronts, covering nine tail seasons of data from four Cluster spacecraft. The probability of finding a whistler in the vicinity of a DF is at least 7 times higher than that of finding it at any random location in the tail box. We find that about $60 \%$ of the 2 min intervals containing a DF were associated with electromagnetic emissions in the whistler frequency range $\left(f_{\mathrm{LH}}-f_{\mathrm{Ce}}\right)$, out of these, at least half had the right-hand polarization consistent with the whistler mode. Therefore, whistlers are a characteristic signature of DFs, suggesting that observation of whistlers together with the characteristic $B_{z}$ signature can be used to identify DFs when no plasma data are available.

We find that whistlers are most often detected in the flux pileup region (FPR) following the DF, close to the center of the current sheet $\left(B_{x} \sim 0\right)$ and in association with electron temperature anisotropy $\left(T_{\perp}>T_{\|}\right)$. Most of the whistlers have small wave normal angles with respect to the background magnetic field. All this suggests that we observe the source region of the emissions and that whistlers are generated by the anisotropic electrons produced by the betatron process inside the FPR. Earlier case studies have shown that whistlers scatter electrons in pitch angle and therefore make a nonadiabatic contribution to otherwise adiabatic (dominated by betatron and Fermi processes) electron dynamics in the FPR. Our statistical results suggest that this scenario could apply to most of the DFs and therefore is of general importance for electron dynamics in the FPR.

Acknowledgments

The data used in this study were obtained from the Cluster Active Archive (CAA) (http://caa.estec.esa. int/). The data sets used are EFW, FGM, PEACE, STAFF, and CIS. Fruitful discussions within the team "Particle Acceleration at Plasma Jet Fronts in the Earth's Magnetosphere," at the ISSI are appreciated. This research is supported by the Swedish National Space Board under grant 135/11:2.

Larry Kepko thanks the reviewers for their assistance in evaluating this paper.

\section{References}

Asano, Y., et al. (2010), Electron acceleration signatures in the magnetotail associated with substorms, J. Geophys. Res., 115, A05215, doi:10.1029/2009JA014587.

Balogh, A., P. J. Cargill, C. M. Carr, M. W. Dunlop, T. S. Horbury, and E. A. Lucek (2001), Magnetic field observations on Cluster: An overview of the first results, in Proceedings of the Les Woolliscroft Memorial Conference / Sheffield Space Plasma Meeting: Multipoint Measurements Versus Theory, edited by B. Warmbein, Eur. Space Agency Spec. Publ., ESA SP-492, 11 pp.

Bortnik, J., J. W. Cutler, C. Dunson, and T. E. Bleier (2007), An automatic wave detection algorithm applied to Pc1 pulsations, J. Geophys. Res., 112, A04204, doi:10.1029/2006JA011900.

Cornilleau-Wehrlin, N., et al. (2003), First results obtained by the Cluster STAFF experiment, Ann. Geophys., 21, 437-456, doi:10.5194/angeo-21-437-2003.

Deng, X., M. Ashour-Abdalla, M. Zhou, R. Walker, M. El-Alaoui, V. Angelopoulos, R. E. Ergun, and D. Schriver (2010), Wave and particle characteristics of earthward electron injections associated with dipolarization fronts, J. Geophys. Res., 115, A09225, doi:10.1029/2009JA015107.

Escoubet, C. P., R. Schmidt, and M. L. Goldstein (1997), Cluster-Science and mission overview, Space Sci. Rev., 79, 11-32, doi:10.1023/A:1004923124586.

Fu, H. S., Y. V. Khotyaintsev, M. André, and A. Vaivads (2011), Fermi and betatron acceleration of suprathermal electrons behind dipolarization fronts, Geophys. Res. Lett., 38, L16104, doi:10.1029/2011GL048528.

Fu, H. S., Y. V. Khotyaintsev, A. Vaivads, M. André, and S. Y. Huang (2012a), Occurrence rate of earthward-propagating dipolarization fronts, Geophys. Res. Lett., 39, L10101, doi:10.1029/2012GL051784. 
Fu, H. S., Y. V. Khotyaintsev, A. Vaivads, M. André, V. A. Sergeev, S. Y. Huang, E. A. Kronberg, and P. W. Daly (2012b), Pitch angle distribution of suprathermal electrons behind dipolarization fronts: A statistical overview, J. Geophys. Res., 117, A12221, doi:10.1029/2012JA018141.

Fu, H. S., Y. V. Khotyaintsev, A. Vaivads, M. André, and S. Y. Huang (2012c), Electric structure of dipolarization front at sub-proton scale, Geophys. Res. Lett., 39, L06105, doi:10.1029/2012GL051274.

Gustafsson, G., et al. (2001), First results of electric field and density observations by Cluster EFW based on initial months of operation, Ann. Geophys., 19, 1219-1240, doi:10.5194/angeo-19-1219-2001.

Huang, S. Y., M. Zhou, X. H. Deng, Z. G. Yuan, Y. Pang, Q. Wei, W. Su, H. M. Li, and Q. Q. Wang (2012), Kinetic structure and wave properties associated with sharp dipolarization front observed by Cluster, Ann. Geophys., 30, 97-107, doi:10.5194/angeo-30-97-2012.

Hwang, K.-J., M. L. Goldstein, E. Lee, and J. S. Pickett (2011), Cluster observations of multiple dipolarization fronts, J. Geophys. Res., 116, A00132, doi:10.1029/2010JA015742.

Khotyaintsev, Y. V., C. M. Cully, A. Vaivads, M. André, and C. J. Owen (2011), Plasma jet braking: Energy dissipation and nonadiabatic electrons, Phys. Rev. Lett., 106, 165001, doi:10.1103/PhysRevLett.106.165001.

Le Contel, O., et al. (2009), Quasi-parallel whistler mode waves observed by THEMIS during near-earth dipolarizations, Ann. Geophys., 27, 2259-2275, doi:10.5194/angeo-27-2259-2009.

Liang, J., B. Ni, C. M. Cully, E. F. Donovan, R. M. Thorne, and V. Angelopoulos (2012), Electromagnetic ELF wave intensification associated with fast earthward flows in mid-tail plasma sheet, Ann. Geophys., 30, 467-488, doi:10.5194/angeo-30-467-2012.

Owen, C. J., et al. (2001), Cluster PEACE observations of electrons during magnetospheric flux transfer events, Ann. Geophys., 19, 1509-1522, doi:10.5194/angeo-19-1509-2001.

Panov, E. V., A. V. Artemyev, W. Baumjohann, R. Nakamura, and V. Angelopoulos (2013), Transient electron precipitation during oscillatory BBF braking: THEMIS observations and theoretical estimates, J. Geophys. Res. Space Physics, 118, 3065-3076, doi:10.1002/jgra.50203.

Pritchett, P. L., and F. V. Coroniti (2011), Plasma sheet disruption by interchange-generated flow intrusions, Geophys. Res. Lett., 38, L10102, doi:10.1029/2011GL047527.

Rème, H., et al. (2001), First multispacecraft ion measurements in and near the Earth's magnetosphere with the identical Cluster ion spectrometry (CIS) experiment, Ann. Geophys., 19, 1303-1354, doi:10.5194/angeo-19-1303-2001.

Santolík, O. (2000), Propagation analysis of STAFF-SA data with coherency tests, Rep. LPCE/NTS/073.A, Lab. Phys. Chimie Environ./CNRS, Orleans, France.

Santolík, O., M. Parrot, and F. Lefeuvre (2003), Singular value decomposition methods for wave propagation analysis, Radio Sci., 38(1), 1010, doi:10.1029/2000RS002523.

Sergeev, V., V. Angelopoulos, S. Apatenkov, J. Bonnell, R. Ergun, R. Nakamura, J. McFadden, D. Larson, and A. Runov (2009), Kinetic structure of the sharp injection/dipolarization front in the flow-braking region, Geophys. Res. Lett., 36, L21105, doi:10.1029/2009GL040658.

Sitnov, M. I., M. Swisdak, and A. V. Divin (2009), Dipolarization fronts as a signature of transient reconnection in the magnetotail, J. Geophys. Res., 114, A04202, doi:10.1029/2008JA013980.

Zhang, Y., H. Matsumoto, and H. Kojima (1999), Whistler mode waves in the magnetotail, J. Geophys. Res., 104, 28,633-28,644, doi:10.1029/1999JA900301.

Zhou, M., M. Ashour-Abdalla, X. Deng, D. Schriver, M. El-Alaoui, and Y. Pang (2009), THEMIS observation of multiple dipolarization fronts and associated wave characteristics in the near-Earth magnetotail, Geophys. Res. Lett., 36, L20107, doi:10.1029/2009GL040663.

Zhou, X.-Z., V. Angelopoulos, V. A. Sergeev, and A. Runov (2010), Accelerated ions ahead of earthward propagating dipolarization fronts, J. Geophys. Res., 115, A00103, doi:10.1029/2010JA015481. 